\title{
EFEITO DA MUSICOTERAPIA SOBRE OS PARÂMETROS VITAIS, ANSIEDADE E SENSAÇÕES VIVENCIADAS NO PERÍODO GESTACIONAL
}

\author{
EFFECT OF MUSIC THERAPY ON VITAL PARAMETERS, \\ ANXIETY AND SENSATIONS EXPERIENCED IN THE \\ GESTATIONAL PERIOD
}

\section{EFECTO DE LA MUSICOTERAPIA EN PARÁMETROS VITALES, ANSIEDAD Y SENSACIONES EXPERIMENTADAS EN EL PERÍODO GESTACIONAL}

\author{
Ana Carolina Almeida Pereira ${ }^{1}$ \\ Viviane Cordeiro de Queiroz ${ }^{2}$ \\ Smalyanna Sgren da Costa Andrade ${ }^{3}$ \\ Ana Carolina Dantas Rocha Cerqueira ${ }^{4}$ \\ Vagna Cristina Leite da Silva Pereira ${ }^{5}$ \\ Simone Helena dos Santos Oliveira ${ }^{6}$
}

Como citar este artigo: Pereira ACA, Queiroz VC, Andrade SSC, Cerqueira ACDR, Pereira VCLS, Oliveira SHS. Efeito da musicoterapia sobre os parâmetros vitais, ansiedade e sensações vivenciadas no período gestacional. Rev baiana enferm. 2021;35:e38825.

Objetivo: avaliar o efeito da musicoterapia sobre os parâmetros vitais, ansiedade e as sensações vivenciadas no período gestacional. Método: estudo de intervenção mista antes e depois, realizado com 30 gestantes atendidas em clínica-escola e no projeto de extensão universitária. Utilizou-se formulário de caracterização sociodemográfica, escala de ansiedade-estado, parâmetros vitais e roteiro de entrevista semiestruturado. Os dados foram analisados por estatística descritiva, inferencial e análise temática de conteúdo. Resultados: houve melhoria da frequência de pulso $(p<0,000)$, respiração $(p=0,002)$, frequência cardíaca $(p<0,000)$ e saturação de oxigênio $(p=0,002)$ evidenciando a efetividade da música sobre estes sinais vitais. Conclusão: a gestação gera possíveis sensações negativas que podem impactar o estado emocional, e a musicoterapia promoveu impacto positivo, pois favoreceu a redução do grau da ansiedade, repercutiu na mobilidade da criança e possuiu efeito significativo na melhoria da pulsação, respiração, frequência cardíaca e saturação de oxigênio.

Descritores: Musicoterapia. Ansiedade. Sinais Vitais. Gestantes. Enfermagem.

Objective: to evaluate the effect of music therapy on the vital parameters, anxiety and sensations experienced during the gestational period. Method: mixed intervention before-after study, conducted with 30 pregnant women who

\footnotetext{
Enfermeira. Especialista em Enfermagem Obstétrica. João Pessoa, Paraíba, Brasil. http://orcid.org/0000-0002-2949- 1988.

Enfermeira. Especialista em Enfermagem Obstétrica. Faculdade Instituto Brasil de Ensino. João Pessoa, Paraíba, Brasil. vivicordeiroqueiroz35@gmail.com. http://orcid. org/0000-0002-2037-92IX

3 Enfermeira. Doutora em Enfermagem. Professora Assistente da Faculdade de Enfermagem Nova Esperança. João Pessoa, Paraíba, Brasil. http://orcid.org/0000-00029812-9376.

4 Enfermeira. Doutora em Enfermagem. Professora da Faculdade de Ciências Sociais Aplicadas. Campina Grande, Paraíba, Brasil. http://orcid.org/0000-000 I-57823102.

Enfermeira. Doutora em Enfermagem. Professora Titular da Universidade Federal da Paraíba. João Pessoa, Paraíba, Brasil. http://orcid.org/0000-0002-8831-3620.

6 Enfermeira. Doutora em Enfermagem. Professora Titular da Universidade Federal da Paraíba. João Pessoa, Paraíba, Brasil. http://orcid.org/0000-0002-9556-I 403.
} 
attended a school clinic and in the university extension project. Sociodemographic characterization form, state anxiety scale, vital parameters and semi-structured interview script were used. The data were analyzed by descriptive statistics, inferential and thematic content analysis. Results: there was an improvement in pulse frequency $(p<0.000)$, breathing $(p=0.002)$, heart rate $(p<0.000)$ and oxygen saturation $(p=0.002)$ evidencing the effectiveness of music on those vital signs. Conclusion: pregnancy generates possible negative sensations that can influence the emotional state, and music therapy promoted a positive impact, as it favored the reduction of the degree of anxiety, had repercussions on the child's mobility and had a significant effect on the improvement of pulse, breathing, heart rate and oxygen saturation.

Descriptors: Music therapy. Anxiety. Vital Signs. Pregnant Women. Nursing.

Objetivo: evaluar el efecto de la musicoterapia en los parámetros vitales, ansiedad y sensaciones experimentadas durante el periodo gestacional. Método: estudio mixto de intervención antes y después, realizado con 30 mujeres embarazadas atendidas en una clínica escolar y en el proyecto de extensión de la universidad. Se utilizó forma de caracterización sociodemográfica, escala de ansiedad del estado, parámetros vitales y guión de entrevista semiestructurado. Los datos fueron analizados mediante estadísticas descriptivas, análisis de contenido inferencial $y$ temático. Resultados: hubo una mejora en la frecuencia del pulso $(p<0.000)$, respiración ( $p=0.002)$, frecuencia cardiaca $(p<0.000)$ y saturación de oxígeno $(p=0.002)$, evidenciándose la eficacia de la música en estos signos vitales. Conclusión: el embarazo genera posibles sensaciones negativas que pueden afectar el estado emocional, y la musicoterapia promovió un impacto positivo, ya que favoreció la reducción del grado de ansiedad, tuvo repercusiones en la movilidad del niño y tuvo un efecto significativo en la mejora del pulso, la respiración, la frecuencia cardiaca y la saturación de oxígeno.

Descriptores: Musicoterapia. Ansiedad. Signos Vitales. Mujeres Embarazadas. Enfermería.

\section{Introdução}

A ansiedade é uma característica prevalente na gestação $^{(1)}$, cujos índices variam de $23 \%$ no Canadá, $15,0 \%$ na Alemanha até 49\% no Paquistão ${ }^{(2)}$. Estudo de coorte no Rio de Janeiro apontou 64,9\% das gestantes com ansiedade ${ }^{(3)}$, prevalência superior aos países desenvolvidos. Para tanto, terapias complementares consistem em recursos acessíveis e efetivos à redução da ansiedade, dentre as quais, a utilização da música e seus elementos, como som, ritmo, melodia e harmonia, produz efeitos emocionais e clínicos benéficos ${ }^{(4)}$.

Não obstante, intervenções musicais mostraram-se eficientes na redução da ansiedade na mulher ${ }^{(5-6)}$ e na melhoria das condições fetais ${ }^{(6-7)}$, constituindo-se alternativa recomendada na área da saúde no âmbito multidisciplinar. Tendo em vista a predisposição da mulher ao sofrimento psíquico no período gestacional, a escolha dessa proposta advém da possibilidade de confirmar a musicoterapia enquanto estratégia de terapia complementar que agrega na prevenção e/ou tratamento de alterações emocionais e parâmetros vitais.

A musicoterapia é uma ferramenta leve de cuidado que pode ser agregada às atividades coletivas do pré-natal. Assim, questiona-se: Qual o impacto do processo gestacional sobre a mulher? A musicoterapia produz respostas emocionais e clínicas satisfatórias? Diante disso, objetiva-se avaliar o efeito da musicoterapia sobre os parâmetros vitais, ansiedade e as sensações vivenciadas no período gestacional.

\section{Método}

Pesquisa do tipo interventiva antes e depois, de abordagem mista, com gestantes atendidas em extensão universitária e clínica-escola pertencentes à instituição de ensino superior de município brasileiro. A amostra por conveniência elegeu 30 gestantes $^{(8)}$ com os seguintes critérios de inclusão: maioridade etária, escolarizadas, com gestação saudável e acima de 20 semanas gestacionais, tempo generalizado na área obstétrica, como o período de desenvolvimento do córtex auditivo, responsável pelo processamento do som. Excluíram-se as mulheres não aptas cognitivo-emocionalmente (autorreferido) e não escolarizadas. 
Os dados foram coletados entre agosto e outubro de 2018, contendo as variáveis de caracterização (naturalidade, idade, situação conjugal, religião, etnia, rendimento mensal, número de filhos, divisão de moradia), sinais vitais da mãe (pulso, respiração, pressão arterial, frequência cardíaca apical, saturação de oxigênio) e do bebê (batimentos cardiofetais).

Além disso, utilizou-se o State-Trait Anxiety Inventory (STAI) validado, adaptado e traduzido para o português como Inventário de Ansiedade Traço-Estado, que apresenta duas escalas: uma, enquanto estado, ou seja, ansiedade momentânea; e outra, enquanto traço, isto é, como parte da constituição psíquica do indivíduo ${ }^{(9)}$.

A escala aplicada neste estudo foi a de ansiedade-estado, ou seja, state (STAI-S) que avaliou como a gestante encontrava-se no momento antes e após a musicoterapia. Em relação ao STAI-S, a escala do tipo Likert de 4 pontos variava de absolutamente não, um pouco, bastante e muitíssimo. A classificação possui graus: baixo (0-34 pontos), moderado (35-49), alto (50-64) e muito alto $(65-80)^{(9)}$.

A pesquisa foi realizada em data e horário previamente estabelecidos, conforme o funcionamento do projeto e da clínica-escola. O ambiente foi reservado com sala climatizada, ornamentada e com pouca luminosidade (presença de luminária), proporcionando ambiência agradável. A coleta foi dividida em cinco etapas, cuja primeira foi o acolhimento da gestante e a aplicação de formulário. Após o contato prévio, no momento da chegada da gestante ao ambiente reservado, houve repetição dos objetivos e da finalidade do estudo e, em seguida, foi aplicado o formulário de caracterização.

A segunda etapa foi a aplicação do STAI-S e aferição dos indicadores vitais. O STAI-S foi entregue para autopreenchimento da gestante antes da intervenção. Após esses procedimentos, que teve a duração média de 10 minutos, foram aferidos os sinais vitais, considerando a necessidade de um período de repouso necessário à verificação dos indicadores das funções vitais $^{(10)}$. Esse tempo foi importante para que os resultados fossem fidedignos e não houvesse interferência do movimento físico sobre os valores mensurados.

O primeiro indicador avaliado foi a saturação de oxigênio $\left(\mathrm{SatO}^{2}\right)$, por meio do oxímetro de pulso de dedo portátil Oximeter, colocando-se o dedo indicador esquerdo da participante no aparelho ${ }^{(11)}$. Após 10 segundos, tempo necessário à leitura conforme instrução do equipamento, foi anotado o nível de saturação.

Com a participante sentada e com o braço apoiado, houve a avaliação do pulso radial, colocando-se os dedos indicador e médio da pesquisadora sobre a artéria radial, pressionando levemente para sentir a pulsação. Quando sentida, foi iniciada a contagem dos batimentos, durante um minuto, observando o ritmo e a frequência. Os valores normais da frequência do pulso são de 60 a 100 batimentos por minuto (BPM) ${ }^{(10)}$. Neste estudo, a estratificação das variáveis foi realizada pela subtração da maior frequência (91 bpm) com a menor (62 bpm), gerando o número primo que permitiu três faixas de intervalos afins.

A respiração foi o próximo indicador avaliado por meio das incursões realizadas. A inspiração e expiração foram contadas como um movimento respiratório. Os valores normais da respiração são de 12 a 20 incursões respiratórias por minuto $(\text { IRPM })^{(10)}$. Para pulso e respiração, foi utilizado um relógio de ponteiro Champion ${ }^{\circledR}$. Ao final de um minuto, estes sinais foram registrados.

A frequência cardíaca foi verificada por meio de um estetoscópio Premium $\AA$, sendo auscultada com atenção, no ponto de impulso máximo, o ritmo cardíaco e a existência de bulhas cardíacas durante um minuto ${ }^{(10)}$. Para avaliação da pressão arterial, foi utilizado o aparelho analógico esfigmomanômetro Premium $\AA$, seguindo a técnica conforme as recomendações sobre o procedimento $^{(10)}$. Na gestante, valores de PAS $\geq 140 \mathrm{mmHg}$ e/ou PAD $\geq 90 \mathrm{mmHg}$ são considerados condizentes com síndromes hipertensiva ${ }^{(11)}$. Os batimentos cardíacos fetais foram avaliados por meio de Sonar digital Baby Doppler International®, durante um minuto, a partir da $12^{\text {a }}$ semana de gestação. A frequência cardíaca fetal é ideal entre 110-160 batimentos por minuto ${ }^{(12)}$. 
$\mathrm{Na}$ terceira etapa realizou-se a primeira entrevista semiestruturada sobre a gestação e as suas repercussões na vida da mulher, para identificação das percepções negativas que podem gerar ansiedade nesse período. Para essa etapa, foi utilizado um gravador de voz, via aplicativo de smartphone. A linha telefônica foi desabilitada momentaneamente na hora da gravação, para que não houvesse nenhuma interrupção durante o processo de entrevista. Os depoimentos apreendidos foram transcritos para compor $\mathrm{O}$ banco de dados.

$\mathrm{Na}$ quarta etapa ocorreu a intervenção por meio da musicoterapia. As músicas foram tocadas em um headphone da marca Philips $@$, sem fio, com Bluetooth, e com entrada para cartão de memória, higienizado a cada uso, com volume autorregulável. A playlist da intervenção foi baseada na música clássica, devido à suavidade, baixa amplitude e simplicidade no ritmo, tocadas sequencialmente até atingir o tempo estimado de 30 minutos, conforme pesquisa similar ${ }^{(13)}$, utilizando-se Sprin Waltz, Nocturne, Four Seasons, Hallelujah - Instrumental (Piano/Violin/Cello Cover), Moonlight Sonata, Waltz of the Flowers. A gestante estava sentada em sofá poltrona reclinável e confortável, com as pernas apoiadas.

A quinta etapa foi a reaplicação das etapas anteriores imediatamente após o término da playlist. A mensuração dos sinais vitais e batimentos cardiofetais foram realizados antes e depois da musicoterapia, seguindo o mesmo padrão descrito anteriormente. As avaliações dos indicadores vitais foram realizadas no mesmo membro pré e pós-intervenção. Foi entregue o STAI-S, para novo autopreenchimento. Após isso, houve outra entrevista, com uso de roteiro semiestruturado sobre a sensação do efeito da música na mãe e como ela sentia o feto.

Cabe ressaltar que para os batimentos cardiofetais houve ausculta no mesmo quadrante da intervenção. Este é o único indicador vital que pode ser avaliado de maneira não invasiva no feto e foi incluído para avaliação do impacto da música sobre a criança, com base no efeito refletido na mãe, tendo em vista que apenas ela ouvia a playlist.
Os dados foram analisados com o Statistical Package for the Social Sciences (SPSS), versão 21. Para as variáveis do estudo foi utilizado o teste de Shapiro-Wilk (amostra<50), que indica a normalidade dos dados para a hipótese testada: a musicoterapia provoca alterações nos parâmetros objeto do estudo. As médias foram comparadas por meio do teste $\mathrm{t}$ pareado devido à natureza do estudo, com $\mathrm{p} \leq 0,05$ para significância estatística.

A transcrição dos depoimentos foi submetida à análise temática de conteúdo ${ }^{(14)}$, que, após as etapas de pré-análise, exploração do material e tratamento dos resultados, derivou em duas categorias temáticas, analisadas à luz do referencial teórico analítico, proposto por Donabedian, e acontece em três etapas (estrutura, processo e resultado); para este estudo foi considerado apenas a terceira etapa, que diz respeito ao resultado $^{(15)}$. Sendo assim, o foco foi verificar o efeito da musicoterapia no período gestacional com base nas sensações percebidas pelas gestantes após a intervenção.

Para preservar o anonimato das gestantes, elas foram codificadas com a letra G (Gestante) e numeradas sequencialmente conforme o atendimento. O estudo seguiu todos os trâmites éticos das pesquisas envolvendo seres humanos da instituição estudada, sob Parecer n. 2.712.697/2018 e Certificado de Apresentação de Apreciação Ética (CAAE): 91072418.1.0000.5179. Cabe ressaltar que o estudo seguiu as recomendações dos guias relacionados ao Consolidated criteria for reporting qualitative research (COREQ) (abordagem qualitativa) e Strengtheningthe Reporting of Observational Studies in Epidemiology (STROBE) (abordagem quantitativa), conferindo maior robustez metodológica.

\section{Resultados}

A maioria das gestantes era natural da capital (63,0\%), com idade média de 26,33 anos $(\mathrm{DP} \pm 5,46)$ e faixa etária em anos entre 24 e $29(37,0 \%)$, com parceiro (77,0\%), evangélica $(57,0 \%)$, de etnia autodeclarada parda $(67,0 \%)$, 
com renda mensal de até um salário mínimo $(57,0 \%)$, primigestas $(60,0 \%)$ e convivendo estritamente com o parceiro $(67,0 \%)$.

Em relação ao pulso, o valor mais próximo ao limiar máximo (100 batimentos por minuto) possuiu 46,0\% de prevalência na pré-intervenção, todavia, as medidas aferidas posteriormente indicaram queda de 13\% nessa avaliação periférica da frequência. No que se refere à respiração, $63,0 \%$ das gestantes possuíam valores abaixo de 15 incursões respiratórias por minuto. A intervenção proporcionou aumento de 20,0\% de prevalência nesse mesmo indicador, atingindo 83\% das participantes, sugerindo possível relaxamento, pois estavam eupnéicas.

O maior valor da pressão arterial sistólica pré-intervenção foi 130 mmHg em 13,0\% das participantes. Após a musicoterapia, este indicador reduziu para 7,0\%. Na pressão arterial diastólica, 7,0\% apresentaram valor com $90 \mathrm{mmHg}$. Após a intervenção, esse indicador foi ausente, havendo contribuição da música para redução na prevalência da pressão arterial (Tabela 1).

Tabela 1 - Monitorização dos sinais vitais das gestantes antes e após a musicoterapia. João Pessoa, Paraíba, Brasil, 2018. (N=30)

\begin{tabular}{|c|c|c|}
\hline \multirow{2}{*}{ Variáveis } & \multicolumn{2}{|c|}{ n (\%) } \\
\hline & Antes & Depois \\
\hline \multicolumn{3}{|c|}{ Pressão arterial sistólica (mmHg) } \\
\hline 90 & $1(3)$ & $1(3)$ \\
\hline 100 & $6(20)$ & $6(20)$ \\
\hline 110 & $5(17)$ & $6(20)$ \\
\hline 120 & $14(47)$ & $15(50)$ \\
\hline 130 & $4(13)$ & $2(7)$ \\
\hline \multicolumn{3}{|c|}{ Pressão arterial diastólica (mmHg) } \\
\hline 50 & $1(3)$ & - \\
\hline 60 & $5(17)$ & $6(20)$ \\
\hline 70 & $10(33)$ & $11(37)$ \\
\hline 80 & $12(40)$ & $13(43)$ \\
\hline 90 & $2(7)$ & - \\
\hline \multicolumn{3}{|c|}{ Frequência de pulso (batimentos por minuto) } \\
\hline $62-70$ & $5(17)$ & $8(27)$ \\
\hline $71-80$ & $11(37)$ & $12(40)$ \\
\hline $81-91$ & $14(46)$ & $10(33)$ \\
\hline \multicolumn{3}{|c|}{ Respiração (incursões respiratórias por minuto) } \\
\hline $12-15$ & $19(63)$ & $25(83)$ \\
\hline $16-20$ & $11(37)$ & $5(17)$ \\
\hline \multicolumn{3}{|c|}{ Frequência cardiaca (batimentos por minuto) } \\
\hline$<60$ & $1(3)$ & $1(3)$ \\
\hline $60-100$ & $26(87)$ & $29(97)$ \\
\hline$>100$ & $3(10)$ & - \\
\hline \multicolumn{3}{|c|}{ Saturação de oxigênio (\%) } \\
\hline Até 97 & $9(30)$ & $1(3)$ \\
\hline $98-100$ & $21(70)$ & $29(97)$ \\
\hline \multicolumn{3}{|c|}{ Batimentos cardíacos fetais (batimentos por minuto) } \\
\hline $110-160$ & $29(97)$ & $30(100)$ \\
\hline$>160$ & $1(3)$ & - \\
\hline
\end{tabular}

Fonte: Elaboração própria.

Nota: Sinal convencional utilizado:

- Dado numérico igual a zero não resultante de arredondamento.

Os resultados foram exitosos, pois as médias da pressão arterial sistólica, diastólica, pulso, respiração, frequência cardíaca materna e fetal mostraram-se reduzidas, ao passo que a saturação de oxigênio aumentou pós-intervenção com a musicoterapia. Neste estudo, embora 
tenha havido redução das médias, estatisticamente a pressão arterial sistólica e diastólica, bem como os batimentos cardiofetais não sofreram influência da intervenção. Houve diferença significativa entre as médias da frequência de pulso $(p<0,000)$, respiração $(p=0,002)$, frequência cardíaca $(p<0,000)$ e saturação de oxigênio $(p=0,002)$ evidenciando a efetividade da música na melhoria destes indicadores vitais (Tabela 2).

Tabela 2 - Médias dos sinais vitais antes e após a musicoterapia. João Pessoa, Paraíba, Brasil, 2018. $(\mathrm{N}=30)$

\begin{tabular}{|c|c|c|c|c|c|c|}
\hline \multirow[b]{2}{*}{ Variáveis } & \multicolumn{2}{|c|}{ Média \pm DP* } & \multirow{2}{*}{$\mathbf{M D i f} \pm \mathbf{D P} \dagger$} & \multirow{2}{*}{ Teste t (gl) $\ddagger$} & \multirow[b]{2}{*}{ p-valor $\$$} & \multirow{2}{*}{ Inf/Sup II } \\
\hline & Antes & Depois & & & & \\
\hline $\begin{array}{l}\text { Pressão } \\
\text { Arterial } \\
\text { Sistólica }\end{array}$ & $114,67 \pm 10,74$ & $113,67 \pm 9,99$ & $1,000 \pm 3,05$ & 1,795 (29) & 0,083 & $-0,139 / 2,139$ \\
\hline $\begin{array}{l}\text { Pressão } \\
\text { Arterial } \\
\text { Diastólica }\end{array}$ & $73,00 \pm 9,52$ & $72,33 \pm 7,73$ & $0,667 \pm 7,39$ & $0,494(29)$ & 0,625 & $-2,095 / 3429$ \\
\hline Pulso & $79,40 \pm 8,62$ & $76,37 \pm 8,57$ & $3,033 \pm 3,54$ & 4,683 (29) & $<0,000$ & $1,709 / 4,358$ \\
\hline Respiração & $14,17 \pm 2,26$ & $13,27 \pm 1,63$ & $0,900 \pm 1,47$ & 3,352 (29) & 0,002 & $0,351 / 1,449$ \\
\hline $\begin{array}{l}\text { Frequência } \\
\text { cardíaca } \\
\text { materna }\end{array}$ & $84,87 \pm 12,29$ & $81,20 \pm 10,09$ & $3,667 \pm 5,02$ & 3,996 (29) & $<0,000$ & $1,790 / 5,544$ \\
\hline $\begin{array}{l}\text { Saturação } \\
\text { de oxigênio }\end{array}$ & $97,77 \pm 0,971$ & $98,33 \pm 0,547$ & $-0,567 \pm 0,93$ & $-3,319(29)$ & 0,002 & $-0,916 / 0,217$ \\
\hline $\begin{array}{l}\text { Batimentos } \\
\text { cardiofetais }\end{array}$ & $139,73 \pm 10,76$ & $138,90 \pm 8,89$ & $0,833 \pm 4,10$ & $1,112(29)$ & 0,275 & $-0,699 / 2,365$ \\
\hline
\end{tabular}

Fonte: Elaboração própria.

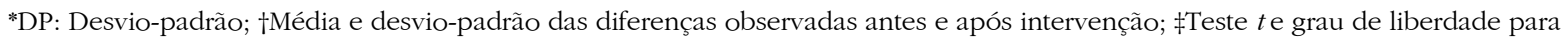
medidas pareadas; §Teste t pareado; II Inf: inferiores/Sup: superiores (nível de confiança de 95\%).

Ainda sobre os resultados descritivos não expostos em tabela, no momento da pré-intervenção, 3\% tinham grau baixo de ansiedade, $87,0 \%$ moderado e 10\% alto. O desfecho após a musicoterapia foi que as mulheres de grau baixo mantiveram-se no mesmo patamar, e 97,0\% das gestantes saíram da estratificação de alto grau para moderado.

Verificou-se que aumentou o percentual dos sentimentos positivos após a intervenção, a exemplo de: calma, segurança, sentimento de estar à vontade, descansada, sentir-se "em casa”, confiança, descontração, satisfação, alegria e sentir-se bem. Houve redução percentual dos sentimentos negativos após a intervenção entre as gestantes, quais sejam: tensão, arrependimento, perturbação, preocupação com possíveis problemas, ansiedade, nervosismo, agitação, sentir-se uma pilha de nervos, preocupação e super agitação (Tabela 3).

Tabela 3 - Sensações antes e após a musicoterapia segundo os itens da escala State-Trait Anxiety Inventory. João Pessoa, Paraíba, Brasil, 2018. (N=30)

(continua)

\begin{tabular}{|c|c|c|c|c|c|c|c|c|}
\hline \multirow{3}{*}{ Itens da escala } & \multicolumn{4}{|c|}{ Antes } & \multicolumn{4}{|c|}{ Depois } \\
\hline & $\mathbf{A N *}$ & $\mathbf{U P} \dagger$ & $\mathbf{B} \ddagger$ & $\S \mathbf{M}$ & $\mathbf{A N}^{*}$ & $\mathbf{U P} \dagger$ & $\mathbf{B} \ddagger$ & $\oint \mathbf{M}$ \\
\hline & n (\%) & n (\%) & n (\%) & n (\%) & n (\%) & n (\%) & n (\%) & n (\%) \\
\hline Calma & $4(13)$ & $12(40)$ & $13(43)$ & $1(4)$ & $4(13)$ & - & $19(63)$ & $7(24)$ \\
\hline Segura & $6(20)$ & $14(46)$ & $9(30)$ & $1(4)$ & - & $12(40)$ & $15(50)$ & $3(10)$ \\
\hline Tensa & $11(37)$ & $13(43)$ & $5(16)$ & $1(4)$ & $19(63)$ & $10(33)$ & $1(4)$ & - \\
\hline
\end{tabular}


Tabela 3 - Sensações antes e após a musicoterapia segundo os itens da escala State-Trait Anxiety Inventory. João Pessoa, Paraíba, Brasil, 2018. (N=30)

(conclusão)

\begin{tabular}{|c|c|c|c|c|c|c|c|c|}
\hline \multirow{3}{*}{ Itens da escala } & \multicolumn{4}{|c|}{ Antes } & \multicolumn{4}{|c|}{ Depois } \\
\hline & $\mathbf{A N}^{*}$ & $\mathbf{U P} \dagger$ & $\mathbf{B} \neq$ & $\S \mathbf{M}$ & AN* & $\mathbf{U P} \dagger$ & $\mathbf{B} \ddagger$ & $\S \mathbf{M}$ \\
\hline & n (\%) & n (\%) & n (\%) & n (\%) & n (\%) & n (\%) & n (\%) & n (\%) \\
\hline Arrependida & $21(70)$ & $6(20)$ & $2(6)$ & $1(4)$ & $25(84)$ & $3(10)$ & $2(6)$ & - \\
\hline À Vontade & $2(6)$ & $18(60)$ & $8(27)$ & $2(7)$ & - & $3(10)$ & $20(67)$ & $7(23)$ \\
\hline Perturbada & $12(40)$ & $12(40)$ & $5(16)$ & $1(4)$ & $21(70)$ & $8(26)$ & $1(4)$ & - \\
\hline Preocupada & $4(13)$ & $18(60)$ & $7(23)$ & $1(4)$ & $5(17)$ & $23(77)$ & $2(6)$ & - \\
\hline Descansada & $16(53)$ & $11(37)$ & $3(10)$ & - & $1(4)$ & $7(23)$ & $18(60)$ & $4(13)$ \\
\hline Ansiosa & $1(4)$ & $8(27)$ & $10(33)$ & $11(36)$ & $6(20)$ & $14(47)$ & $9(30)$ & 1(3) \\
\hline Sinto "em casa" & $8(27)$ & $11(37)$ & $9(30)$ & $2(6)$ & $1(4)$ & $10(33)$ & $13(43)$ & $6(20)$ \\
\hline Confiante & $2(6)$ & $15(50)$ & $7(24)$ & $6(20)$ & - & $6(20)$ & $16(53)$ & $8(27)$ \\
\hline Nervosa & $3(10)$ & $12(40)$ & $10(33)$ & $5(17)$ & $16(53)$ & $13(43)$ & $1(4)$ & - \\
\hline Agitada & $10(33)$ & $11(37)$ & $5(17)$ & $4(13)$ & $22(73)$ & $8(27)$ & - & - \\
\hline Pilha de nervos & $9(30)$ & $14(47)$ & $5(17)$ & $2(6)$ & $21(70)$ & $8(27)$ & - & $1(3)$ \\
\hline Descontraída & $9(30)$ & $14(47)$ & $6(20)$ & $1(3)$ & - & $5(17)$ & $21(70)$ & $4(13)$ \\
\hline Satisfeita & $2(6)$ & $11(37)$ & $11(37)$ & $6(20)$ & - & $3(10)$ & $18(60)$ & $9(30)$ \\
\hline Preocupada & $5(17)$ & $21(70)$ & $3(10)$ & $1(3)$ & $9(30)$ & $2(70)$ & - & - \\
\hline Agitada e confusa & $9(30)$ & $15(50)$ & $5(17)$ & $1(3)$ & $25(83)$ & $3(10)$ & $2(7)$ & - \\
\hline Alegre & $4(13)$ & $7(24)$ & $13(43)$ & $6(20)$ & - & $1(3)$ & $12(40)$ & $17(57)$ \\
\hline Bem & $2(7)$ & $13(43)$ & $7(23)$ & $8(27)$ & - & $1(3)$ & $11(37)$ & $18(60)$ \\
\hline
\end{tabular}

Fonte: Elaboração própria.

Nota: Sinal convencional utilizado:

- Dado numérico igual a zero não resultante de arredondamento.

* $\mathrm{AN}=$ Absolutamente não. $\nmid \mathrm{UP}=\mathrm{Um}$ pouco. $\ddagger \mathrm{B}=$ Bastante. $₫ \mathrm{M}=$ Muitíssimo.

A abordagem qualitativa foi necessária para compreender as falas frente à ansiedade no período gestacional, antes e após a intervenção musical, permitindo a construção de categorias geradas com base nos questionamentos: A sua gestação foi planejada? Como foi a sua reação ao descobrir? Que sentimento representa o que você está sentindo hoje? Qual a sensação gerada pela música? Como percebeu o seu bebê enquanto você ouvia música?

\section{A descoberta da gravidez e o processo gestacional como possiveis geradores de sensações negativas}

Embora a descoberta traga reações sentimentais diferenciadas, a revelação dessa nova condição gerou sensação de espanto, além de medo, ansiedade e oscilação emocional frente à gestação:

Quando eu descobri, eu fiquei em choque. (G1).

No começo dá um susto, mas depois vem a alegria. (G17).

Fico com medo... pensando que vai acontecer algo. (G3).

Seis meses tentando, era muita ansiedade, pareceu que tinha sido anos. (G2).

O lado ruim é pela ansiedade. (G10).

Percebeu-se nas falas das mulheres o receio e a insegurança quanto ao futuro não controlável e imprevisível, o que pode gerar estado de ansiedade. A notícia da gravidez traz impacto, pois os sentimentos inerentes à gestação não estão associados somente ao bebê:

A responsabilidade, a espera e não saber se vou dar conta. (G3). 
Fiquei preocupada. (G6).

Hoje me sinto mais tranquila e estável, porém, ainda com receio do futuro. (G18).

Às vezes fico pensando se vou dar conta ou não, se vou saber criar. (G29).

Cabe ressaltar que o planejamento do momento adequado e o parceiro certo para engravidar repercutem no estado emocional das gestantes, enfatizando a importância da rede de apoio para redução do risco de sentimentos negativos geradores da ansiedade:

Ouvi palavras negativas de pessoas que eu amo. (G2).

No primeiro momento foi um desespero e tristeza. Me sinto um pouco só, isso é o que me deixa triste, não tenho o devido apoio e alicerce que gostaria e que preciso. (G18).

Me faz sentir triste é o que os outros fala comigo, a maneira que eles falam. (G25).

Tem vezes que a gente escuta muita coisa ruim dos outros e isso acaba deixando a gente pra baixo. (G28).

Musicoterapia como geradora de sensações positivas na mãe e reativas no feto.

Após a seção de musicoterapia, as mulheres foram questionadas quanto às sensações geradas no pós-intervenção. De acordo com os depoimentos, ouvir música gera sensação de paz, força, leveza, tranquilidade e conexão da mente e do corpo, ouvir e cantar música são comportamentos que proporcionam boa expressividade emocional:

Tranquilidade, paz e sossego. (G1, G3, G28).

Alivio. (G2).

Emoção. (G4, G22).

Paz. (G4, G26-G27, G29-G30).

Calmaria. (G17).

Sensação de alegria. (G19).

Gratidão e paz. (G5).

Alegre. (G8, G9).

Tranquila.(G1, G6, G8-G9, G11-G12, G14, G17, G21-G22, G26-G27, G29-G30).

Cura interior. (G16).

Me sinto mais feliz. (G28).

Sinto uma paz na alma. (G16).

Me sinto leve. (G1, G6, G17, G21-G22).

Me sinto calma. (G2-G3, G5, G10, G12, G17, G20, G23, G25-G30).
Sinto uma fortaleza. (G5).

Feliz. (G7-G8, G14, G18, G22-G23, G25).

Descansada e desligada do mundo. (G24).

Esqueço os problemas. (G28).

Me sinto mais alegre, calma e feliz, começo a cantar também. (G30).

Me sinto bem. (G1, G6, G10, G18-G20, G22).

Embora apenas a gestante tivesse ouvido a playlist por meio dos headphones, a música provocou reações diferenciadas de mobilidade fetal, segundo as falas:

Ficou quietinho. (G4, G8-G10, G12-G13, G25, G27, G30).

Ficou tranquilo. (G7, G11, G22-G23).

Ele ficou calmo, é como a gente tá, se eu ficar agitada ele também fica, ele sente tudo. (G5).

Ele ficou calmo, mas na de Beethoven mexeu mais. (G3).

Ele ficou mexendo um pouco. (G1).

Mexeu bastante. (G2, G6, G16-G17, G21, G24, G26, G28-G29).

\section{Discussão}

De acordo com os resultados, as médias dos parâmetros vitais mostraram-se melhorados após a intervenção musical, conducentes com sensação de relaxamento. A Enfermagem pode incorporar em suas práticas, o uso de instrumentos multidisciplinares para a identificação dos níveis de ansiedade considerando a natureza causal do processo gestacional, além de estratégias complementares, como a musicoterapia, para fornecer cuidado na redução de riscos clínicos decorrentes de alterações nos sinais vitais. A música é uma tecnologia eficaz, não farmacológica e de baixo custo, o que torna o estudo relevante para as mulheres e a equipe multiprofissional.

Estudos têm evidenciado o efeito da musicoterapia contribuindo para a manutenção de valores satisfatórios de frequência cardíaca ${ }^{(7,16)}$, $\operatorname{respiração~}^{(4,7)}$, pressão $\operatorname{arterial}^{(4,7,16)}$ e pulso ${ }^{(4,16)}$, o que reforça os dados deste estudo. A melhoria de todos os percentuais com escala de resposta satisfatória nos itens do STAI-S demonstra a influência do som sobre a percepção de estímulo à calmaria, segurança, descanso, confiança, alegria e bem-estar, bem como na redução da tensão, 
preocupação, perturbação, ansiedade e nervosismo, corroborando pesquisas nacional e internacional sobre a efetividade da estratégia musical na redução da ansiedade ${ }^{(4-5,7,16)}$.

Sobre as falas, insegurança e ansiedade podem estar relacionadas ao apoio emocional durante a gestação ${ }^{(2,17-19)}$. Além disso, hesitação, instabilidade, responsabilidade e o cuidado voltado ao novo ser podem ser sensações inerentes à gravidez. Por isso, sentimentos negativos podem levar a reações emocionais exacerbadas e desfechos perinatais desfavoráveis ${ }^{(20-21)}$, podendo causar sofrimento psíquico ${ }^{(22)}$. A adesão às terapias complementares é uma alternativa de prevenção ao adoecimento mental em gestantes ${ }^{(23)}$, cuja musicoterapia proporciona desligamento e reflexão, melhorando o bem-estar biopsicossocial $^{(22,24)}$.

Embora neste estudo a música clássica tenha sido o estilo selecionado para manter o rigor da intervenção, a redução da ansiedade depende da melodia escolhida pelo indivíduo que se conecta com o ritmo que mais lhe agrada ${ }^{(13)}$, favorecendo a espiritualização e se constituindo como ferramenta útil à autocura das aflições e anseios no período gestacional.

Sobre o efeito da música sobre o feto, embora não tenha havido significância estatística no pré e pós-teste, a média dos batimentos cardiofetais reduziu, além de nenhum feto ter se encontrado com taquicardia pós-intervenção. As falas mostraram o reflexo da musicoterapia na mobilidade da criança e na conexão da mãe com o bebê, podendo favorecer a construção do vínculo afetivo. Dessa forma, músicas ouvidas na gestação podem impactar o bebê após o nascimento, melhorando a ambiência, o sono e a redução da frequência do choro ${ }^{(25)}$.

Com base no referencial teórico adotado para avaliação do resultado, a qualidade do cuidado pode ser indicado pelos riscos, benefícios, custos e acesso ao serviço prestado. Mais precisamente, a qualidade é mensurada pelo efeito causado nos indivíduos ${ }^{(15)}$. Articulando os conceitos com os achados deste estudo, a musicoterapia é um recurso terapêutico eficaz a ser utilizado no período gestacional, sendo evidenciado que contribuiu positivamente para melhoria dos aspectos clínicos e da saúde mental da grávida, e pode reverberar na saúde fetal, conforme indicado pela literatura. Após a intervenção verificou-se o benefício da terapia, devendo ser repensadas estratégias para a efetivação dessa prática na assistência pré-natal, uma vez que, fazer uso dos recursos técnicos disponíveis na assistência à saúde, minimiza os riscos, levando a mudanças favoráveis no processo de cuidado individual e coletivo.

O não uso de dispositivos eletrônicos para comparação da acurácia das medidas e a utilização de um estilo musical com diversos ritmos constituíram-se como limitações deste estudo. Sugerem-se uso de protocolos controlados à identificação fidedigna das medidas e das notas musicais atuantes sobre as variáveis.

\section{Conclusão}

A gestação é uma possível condição geradora de sensações negativas que podem impactar $\mathrm{O}$ estado emocional. A musicoterapia promoveu efeitos positivos, favoreceu a redução do grau da ansiedade, repercutiu na mobilidade da criança e possuiu efeito significativo na melhoria da pulsação, respiração, frequência cardíaca e saturação de oxigênio, sem impactar os batimentos cardiofetais e as pressões arteriais sistólica e diastólica.

\section{Colaborações:}

1 - concepção, projeto, análise e interpretação dos dados: Ana Carolina Almeida Pereira e Smalyanna Sgren da Costa Andrade;

2 - redação do artigo e revisão crítica relevante do conteúdo intelectual: Ana Carolina Almeida Pereira, Viviane Cordeiro de Queiroz e Smalyanna Sgren da Costa Andrade;

3 - aprovação final da versão a ser publicada: Ana Carolina Almeida Pereira, Viviane Cordeiro de Queiroz, Smalyanna Sgren da Costa Andrade, Ana Carolina Dantas Rocha Cerqueira, Vagna Cristina Leite da Silva Pereira e Simone Helena dos Santos Oliveira. 


\section{Referências}

1. Silveira RAM, Grossi-Milani R, Velho APM, Marques AG. Perception of pregnant women about self-care and maternal care. Rev Rene. 2016;17(6):758-65. DOI: 10.15253/2175-6783.2016 000600005

2. Silva MMJ, Nogueira DA, Clapis MJ, Leite EPRC. Anxiety in pregnancy: prevalence and associated factors. Rev esc enferm USP. 2017;51:e03253. DOI: 10.1590/S1980-220X 2016048003253

3. Morais AODS, Simões VMF, Rodrigues LDS, Batista RFL, Lamy ZC, Carvalho CA, et al. Maternal depressive symptoms and anxiety and interference in the mother/child relationship based on a prenatal cohort: an approach with structural equations modeling. Cad Saúde Pública. 2017;33(6):e00032016. DOI: 10.1590/0102-311X00032016

4. Melo GAA, Rodrigues AB, Firmeza MA, Grangeiro ASM, Oliveira PP, Caetano JÁ. Musical intervention on anxiety and vital parameters of chronic renal patients: a randomized clinical trial. Rev Latino-Am Enfermagem. 2018;26:e2978. DOI: 10.1590/1518-8345.2123.2978

5. Lin CJ, Chang YC, Chang YH, Hsiao YH, Lin HH, Liu SJ, et al. Music interventions for anxiety in pregnant women: a systematic review and meta-analysis of randomized controlled trials. J Clin Med. 2019;8(11):1884. DOI: 10.3390/jcm8 111884

6. Garcia-Gonzalez J, Ventura-Miranda MI, Requena-Mullor M, Parron-Carreño $\mathrm{T}$, Alarcon-Rodriguez R. State-trait anxiety levels during pregnancy and foetal parameters following intervention with music therapy. J Affect Disord. 2018;232:17-22. DOI: 10.1016/j.jad. 2018.02.008

7. Gonzaléz JG, Miranda MIV, Garcia FM, Ruiz TIP, Gascón MLM, Mullor MR, et al. Effects of Prenatal Music Stimulation on Fetal Cardiac State, Newborn Anthropometric Measurements and Vital Signs of Pregnant Women: A Randomized Controlled Trial. Complement Ther Clin Pract. 2017;27:61-7. DOI: 10.1016/j. ctcp.2017.03.004

8. Triola MF. Introdução à Estatística - Atualização da Tecnologia. 11a ed. Rio de Janeiro: LTC; 2013.

9. Biaggio A, Natalício L. Manual para o Inventário de Ansiedade Traço-Estado (IDATE). Rio de Janeiro: CEPA; 1979.
10. Jensen S. Semiologia para Enfermagem - Conceitos e Prática Clínica. Rio de Janeiro: Guanabara Koogan; 2013.

11. Sociedade Brasileira de Pneumologia e Tisiologia. Oximetria de Pulso [Internet]. Brasília (DF); 2018 [cited 2020 Jan 12]. Available from: https://sbpt.org.br/portal/espacosaude-respiratoria-oximetria-de-pulso/

12. Brasil. Ministério da Saúde. Instituto Sírio-Libanês de Ensino e Pesquisa. Protocolos da Atenção Básica: Saúde das Mulheres [Internet]. Brasília (DF); 2016 [cited 2020 Jan 22]. Available from: http://bvsms.saude.gov.br/bvs/_ publicacoes/ protocolos_atencao_basica_saude_mulheres.pdf

13. Zhou K, Li X, Li J, Liu M, Dang S, Wang D, et al. A clinical randomized controlled trial of music therapy and progressive muscle relaxation training in female breast cancer patients after radical mastectomy: results on depression, anxiety and length of hospital stay. Eur J Oncol Nurs. 2015;19(1):54-9. DOI: 10.1016/j.ejon.2014.07.010

14. Taquette SR, Minayo MC. An analysis of articles on qualitative studies conducted by doctor published in scientific journals in Brazil between 2004 and 2013. Physis. 2017;26(2):417-34. DOI: 10.1590/s0103-73312017000200010

15. Donabedian A. Explorations in Quality Assessment and Monitoring. Michigan (US): Health Administration Press; 1980.

16. Betegon AA, García M, Parés S, Montenegro G, Feixas G, Padilla N, et al. A Program Aimed at Reducing Anxiety in Pregnant Women Diagnosed With a Small-for-Gestational-Age Fetus: Evaluative Findings From a Spanish Study. J Perinatal Neonatal Nurs. 2017;31(3):225-35. DOI: 10.1097/ JPN.0000000000000270

17. Rees S, Channon S, Waters CS. The impact of maternal prenatal and postnatal anxiety on children's emotional problems: a systematic review. Eur Child Adolesc Psychiatry. 019;28(2):257-80. DOI: 10.1007/s00787-018-1173-5

18. Sujan AC, Rickert ME, Oberg AS, Quinn PD, Hernández-Díaz S, Almqvist C, et al. Associations of Maternal Antidepressant Use During the First Trimester of Pregnancy With Preterm Birth, Small for Gestational Age, Autism Spectrum Disorder, and Attention-Deficit/Hyperactivity Disorder in Offspring. Jama. 2017;317(15):1553-62. DOI: 10.1001/ jama.2017.3413

19. Freeman MP, Góez-Mogollón L, Mcinerney KA, Davies AC, Church TR, Sosinsky AZ, et al. Obstetrical 
and neonatal outcomes after benzodiazepine exposure during pregnancy: Results from a prospective registry of women with psychiatric disorders. Gen Hosp Psychiatry. 2018;53:73-9. DOI: 10.1016/j.genhosppsych.2018.05.010

20. Corbijn van Willenswaard K, Lynn F, McNeill J, McQueen K, Dennis CL, Lobel M, et al. Music interventions to reduce stress and anxiety in pregnancy: a systematic review and meta-analysis. BMC Psychiatry. 2017;17(1):271. DOI: $10.1186 /$ s12888-017-1432-x

21. Lima MOP, Tsunechiro MA, Bonadio IC, Murata M. Depressive symptoms in pregnancy and associated factors: longitudinal study. Acta paul enferm. 2017;30(1):39-46. DOI: 10.1590/1982-0194201700007

22. Fancourt D, Perkins R. Could listening to music during pregnancy be protective against postnatal depression and poor wellbeing post birth? Longitudinal associations from a preliminary prospective cohort study. BMJ Open. 2018;8(7):e021251. DOI: 10.1136/ bmjopen-2017-021251
23. Van Ravesteyn LM, Lambregtse - van den Berg MP, Hoogendijk WJG, Kamperman AM. Interventions to treat mental disorders during pregnancy: a systematic review and multiple treatment metaanalysis. PLoS One. 2017;12(3):e0173397. DOI: 10.1371/journal.pone.0173397

24. Hepp P, Hagenbeck C, Gilles J, Wolf OT, Goertz W, Janni W, et al. Effects of music intervention during caesarean delivery on anxiety and stress of the mother a controlled, randomised study. BMC Pregnancy Childbirth. 2018;18(1):435. DOI: 10.1186/s12884-018-2069-6

25. van der Heijden MJE, Oliai Araghi S, Jeekel J, Reiss IKM, Hunink MGM, van Dijk M. Do Hospitalized Premature Infants Benefit from music Interventions? A Systematic Review of Randomized Controlled Trials. PloS One. 2016;11(9):e0161848. DOI: 10.1371 journal. pone. 0161848

Recebido: 16 de setembro de 2020

Aprovado: 4 de março de 2021

Publicado: 31 de março de 2021

A Revista Baiana de Enfermagem utiliza a Licença Creative Commons - Atribuição-NãoComercial 4.0 Internacional. https://creativecommons.org/licenses/by-nc/4.0/ Este artigo é de acesso aberto distribuído sob os termos da Licença Creative Commons (CC BY-NC). Esta licença permite que outros remixem, adaptem e criem a partir do seu trabalho para fins não comerciais. Embora os novos trabalhos tenham de lhe atribuir o devido crédito e não possam ser usados para fins comerciais, os usuários não têm de licenciar esses trabalhos derivados sob os mesmos termos. 